Applied Physiology, Nutrition, and Metabolism Physiologie appliquée, nutrition et métabolisme

\title{
Impaired handgrip exercise-induced brachial artery flow- mediated dilation in young obese males
}

\begin{tabular}{|r|l|}
\hline Journal: & Applied Physiology, Nutrition, and Metabolism \\
\hline Manuscript ID & apnm-2015-0459.R1 \\
\hline Manuscript Type: & Article \\
\hline Complete List of Authors: & $\begin{array}{l}\text { Slattery, David; Queen's University, School of Kinesiology and Health } \\
\text { Studies } \\
\text { Stuckless, Troy; Queen's University, School of Kinesiology and Health } \\
\text { Studies } \\
\text { King, Trevor; Queen's University, School of Kinesiology and Health Studies } \\
\text { Pyke, Kyra; Queen's University, School of Kinesiology and Health Studies }\end{array}$ \\
\hline Keyword: & \begin{tabular}{l} 
shear stress, endothelial function, FMD, reactive hyperemia, obesity \\
\hline
\end{tabular} \\
\hline
\end{tabular}

SCHOLARONE ${ }^{\text {m }}$

Manuscripts 
Impaired handgrip exercise-induced brachial artery flow-mediated dilation in young obese males

\section{David J. Slattery, Troy J.R. Stuckless, Trevor J. King and Kyra E. Pyke}

Address Correspondence To:

Dr. Kyra E. Pyke, PhD

Associate Professor

Cardiovascular Stress Response Laboratory

School of Kinesiology and Health Studies

Queen's University

Kingston, ON

K7L 3N6

Tel: (613) 533-6000, x79631

Fax: (613) 533-2009

E-mail: pykek@queensu.ca 


\begin{abstract}
PURPOSE: Flow mediated dilation (FMD) stimulated by different shear stress stimulus profiles may recruit distinct transduction mechanisms, and provide distinct information regarding endothelial function. The purpose of this study was to determine whether obesity influences brachial artery FMD differently depending on the shear stress profile used for FMD assessment. METHODS: The FMD response to a brief, intermediate and sustained shear stress profile was assessed in obese $(n=9)$ and lean $(n=19)$ young men as follows: Brief stimulus- standard reactive hyperemia $(\mathrm{RH})$ following a $5 \mathrm{~min}$ forearm occlusion ( $5 \mathrm{~min} \mathrm{RH}$ ); Intermediate stimulus- $\mathrm{RH}$ following a $15 \mathrm{~min}$ forearm occlusion (15 min RH); Sustained stimulus- $10 \mathrm{~min}$ of handgrip exercise (HGEX). Brachial artery diameter and mean shear stress were assessed using echo and Doppler ultrasound respectively during each FMD test. RESULTS: There was no group difference in HGEX shear stress $(\mathrm{p}=0.390)$ however, the obese group had a lower HGEX-FMD $(5.2 \pm 3.0 \%$ vs. $11.5 \pm 4.4 \%, \mathrm{p}<0.001)$. There was no group difference in $5 \mathrm{~min}$ RH-FMD $(\mathrm{p}=0.466)$ or $15 \mathrm{~min}$ RH-FMD $(\mathrm{p}=0.181)$, however the shear stress stimulus was larger in the obese group. After normalization to the stimulus the $15 \mathrm{~min}$ RH-FMD ( $\mathrm{p}=0.002)$, but not the 5 min RH-FMD ( $p=0.118)$ was lower in the obese group. CONCLUSION: These data suggest that obesity may have a more pronounced impact on the endothelium's ability to respond to prolonged increases in shear stress.
\end{abstract}

KEYWORDS - Shear stress, endothelial function, FMD, reactive hyperemia, obesity 


\section{Introduction}

The endothelium plays an integral role in maintaining vascular health (Deanfield et al. 2007) and endothelial function can be assessed non-invasively by measuring the magnitude of endothelial dependent dilation following an experimenter imposed increase in blood flow associated shear stress (flow mediated dilation; FMD) (Celermajer et al. 1992). The standard methodology used to assess FMD is the reactive hyperemia test, which stimulates a large but transient increase in conduit artery shear stress via the release of a 5 minute limb occlusion (5 min RH-FMD) (Celermajer et al. 1992). This test has been shown to have some utility in predicting future risk of cardiovascular events in both disease and healthy populations (Inaba et al. 2010; Thijssen et al. 2011). However, there is growing evidence to suggest that other shear stress profiles elicit different transduction mechanisms and that the resulting FMD response could provide distinct or complementary information regarding the function of the endothelium (Findlay et al. 2013;Frangos et al. 1996;Mullen et al. 2001;Szijgyarto et al. 2013). Thus inclusion of more than one unique shear stress profile when investigating FMD may allow a more comprehensive characterization of endothelial function.

Lengthening the duration of occlusion can prolong the shear stress stimulus created by reactive hyperemia. This typically yields a larger FMD response than the standard 5 min-RH test (Ku et al. 2014; Mullen et al. 2001), and has been minimally studied with respect to its ability to detect endothelial dysfunction in a variety of populations (Mullen et al. 2001). Handgrip exercise (HGEX) elicits a sustained, intensity-dependent increase in brachial artery shear stress (Findlay et al. 2013;Pyke et al. 2008b; Wray et al. 2006). Compared to an RH profile this better reflects how shear stress increases in response to daily activity. Exercise induced FMD (EX-FMD) may also play a role in perfusion (Duffy et al. 1999; Nabel et al. 1990; Vita and Hamburg 2010), therefore it is important that the factors that influence this response are fully understood. Recent evidence suggests that HGEX stimulated FMD (HGEX-FMD) vs. 5 min RH-FMD may be impacted differently by acute stimuli (Padilla et al. 2006;Szijgyarto et al. 
2013) and that HGEX-FMD is more profoundly impacted by a short history of smoking (Findlay et al. 2013). Collectively this indicates that 1) 5 min RH-FMD responses cannot be generalized to HGEX-FMD and 2) HGEX-FMD may be an indicator of emerging endothelial dysfunction (Bellien et al. 2010; Findlay et al. 2013). Thus, HGEX-FMD may reveal endothelial dysfunction that is not captured by a standard 5 min RH-FMD test.

Many (Arcaro et al. 1999;Brook et al. 2001; Meyer et al. 2006;Olson et al. 2006; Woo et al. 2004), but not all (Biasucci et al. 2010), studies have reported impaired 5 min RH-FMD in obese vs. healthy weight populations, and it is thought that endothelial dysfunction may provide an important mechanistic link between obesity and increased risk of cardiovascular disease (Van Gaal et al. 2006). However, no studies to date have examined the ability of the arteries to respond to more sustained shear stress stimuli in an obese population, and this would provide more detailed information regarding the extent or nature of endothelial dysfunction. The purpose of the present study was therefore to compare FMD between young, obese participants vs. lean controls in response to three distinct shear stress stimulus profiles ( 5 min RH, 15 min RH and HGEX). We hypothesized that FMD would be lower in all tests in the obese participants, and, in agreement with our findings in young smokers (Findlay et al. 2013), that the most pronounced difference between the groups would be found for the HGEX-FMD response.

\section{Methods}

\section{Participants}

Nine obese males (aged 18-40) and nineteen healthy-weight, control subjects participated. The study protocol was approved by the Health Sciences Research Ethics Board at Queen's University, and participants provided written informed consent to participate on forms approved by this board.

In order to be included in the obese group, participants were required to have a waist circumference of $\geq 102 \mathrm{~cm}$ (40 inches). In order to be included in the control group, participants were required to have a body mass index (BMI) that was less than $25 \mathrm{~kg} / \mathrm{m}^{2}$ and a waist circumference of $\leq 86$ cm (34 inches) (Han 1995). All participants were non-smokers, not engaged in any regular structured 
physical activity, and were free from any known cardiovascular disease. Hypertensive individuals (hypertension defined as systolic $\geq 140 \mathrm{mmHg}$ and diastolic $\geq 90 \mathrm{mmHg}$ ) and individuals taking medications related to cardiovascular risk factor control (i.e. to control plasma lipid, glucose levels or blood pressure) were excluded.

In an initial visit participants were screened for anthropometric measurements, medical history and blood pressure (screened via a BpTRU device; Medical Devices, Coquitlam, British Columbia, Canada). They were then familiarized with the study equipment and protocol. Participants were instructed to abstain from alcohol, caffeine, and food for 12 hours and from exercise for 24 hours prior to the single experimental visit. Visits occurred in the morning (starting between 8 am - 9:30 am) in a quiet temperature controlled room $\left(20.5^{\circ} \mathrm{C}-23^{\circ} \mathrm{C}\right)$.

\section{Experimental Protocol}

Upon arriving at the laboratory, participants were asked to lie in a supine position for 30 minutes. During the rest period, they completed a 7-day physical activity questionnaire to estimate physical activity levels. Blood was drawn for determination of blood viscosity, glucose and lipids. At the end of this initial rest period arterial stiffness (via pulse wave velocity (PWV)) and common carotid artery intima media thickness (IMT) were assessed to provide a thorough characterization of vascular health. Participants then completed five FMD trials: two consecutive HGEX trials, two consecutive 5 min occlusion RH trials, and one 15 min occlusion RH trial. The order in which the HGEX and 5 min RH trials occurred was counterbalanced (i.e. whether the two 5 min RH or the two HGEX trials were performed first was rotated between participants), while the $15 \mathrm{~min}$ RH test was always the final test of the session. A minimum of 10 minutes, or until baseline diameter was re-established elapsed between trials. Figure 1 outlines the experimental protocol. The stability of RH-FMD (Harris et al. 2006;Pyke and Jazuli 2011) and HGEX-FMD (Pyke and Jazuli 2011) responses over several closely spaced trials has been demonstrated previously.

Heart Rate and Blood Pressure monitoring 
Heart rate (HR) was monitored with a three-lead electrocardiogram for the duration of the study. Mean arterial pressure was monitored throughout each trial via photoplethysmography (Finometer PRO; Finapres Medical Systems, Amsterdam, The Netherlands). To achieve this a pneumatic finger cuff was secured around the right middle finger with the hand placed at heart level.

\section{Brachial artery blood velocity and diameter measurement}

An ultrasound probe (Vivid i2; GE Medical Systems, London, ON, Canada) was placed over the left brachial artery to acquire an optimal image of the vessel and a clear arterial blood velocity signal. Once in position, a guide was secured to the skin so that the same region of the vessel was scanned in all trials. For reasons previously described, all scans were performed at an insonation angle of $68^{\circ}$ (Pyke et al. 2008a). The brachial artery image was measured with the ultrasound probe operating at $12 \mathrm{MHz}$. The image was captured using a VGA to USB frame grabber (Epiphan systems Inc.) and was recorded in .avi format on an external computer with commercially available software (Camtasia Studio; TechSmith, Okemos, MI, USA) for subsequent diameter analysis. The brachial artery mean blood velocity was measured with Doppler ultrasound operating at $4 \mathrm{MHz}$ (GE Vivid i2; GE Medical Systems, London, ON, Canada). The Doppler shift frequency spectrum was analyzed using a Multigon 500P TCD (Multigon Industries, Yonkers, NY, USA). The corresponding voltage output was sampled continuously (Powerlab; AD Instruments, Colorado Springs, CO, USA) and stored (LabChart; AD Instruments) for later analysis.

\section{Blood Viscosity}

Blood was drawn from a vein in the antecubital region prior to the beginning of data collection and was promptly analyzed at a shear rate of $225 \mathrm{~s}^{-1}$ (Brookfield Viscometer DV-II+ Pro) at $37 \pm 2^{\circ} \mathrm{C}$. The venous blood sample was not obtained in 3 participants (obese: $n=2$; control: $n=1$ ).

Blood Lipid and Glucose Analysis

A small sample of the venous blood was used to analyze fasting blood lipid and glucose levels using a Cholestech LDX System (Alere Inc., Ottawa, ON, Canada). 


\section{Handgrip exercise flow-mediated dilation test (HGEX-FMD)}

Participants extended their left arm and a handgrip device was placed in their left hand. The real time blood velocity was displayed on a computer screen as a 6 second moving average. Following 1 minute of baseline recordings, handgrip exercise was initiated at the intensity (established in the initial familiarization visit) that achieved the required blood velocity for a shear stress target of $17.5 \mathrm{dyn} / \mathrm{cm}^{2}$. The blood velocity required to achieve the target shear stress was calculated for each participant as:

Required Velocity $=17.5 \mathrm{dyn} / \mathrm{cm}^{2} \times$ brachial artery diameter $/ 4 x$ Viscosity

In the cases $(n=3)$ where blood viscosity was not obtained, the group mean was used in its place. The participant received visual feedback regarding their exercise intensity by displacing a force output line on a computer screen to a target controlled by an experimenter (Pyke et al. 2008b). Contractions were performed in a $1 \mathrm{~s}$ contraction, $5 \mathrm{~s}$ relaxation pattern (King et al. 2013). Participants were coached through minor increases and decreases in contraction intensity in order to maintain the required blood velocity for the 10 minute exercise bout.

\section{Reactive hyperemia flow-mediated dilation tests (RH-FMD)}

Participants extended their left arm and an occlusion cuff was secured around their forearm immediately distal to the antecubital fossa. Baseline recordings were made for 1 minute. The occlusion cuff was then inflated to a pressure of $250 \mathrm{mmHg}$ for a period of 5 minutes or 15 minutes. Recording of blood velocity and vessel diameter resumed $1 \mathrm{~min}$ prior to cuff deflation and continued for a further 3 minutes post-deflation.

\section{Common Carotid artery IMT}

IMT was measured by applying a linear ultrasound probe operating at $12 \mathrm{MHz}$ in B-mode (Vivid i2; GE Medical Systems, London, ON, Canada) to the left common carotid artery, such that the carotid sinus was visible. This ensured that the image was taken at a similar location in all subjects. Each recording lasted for a minimum of 10 seconds. Carotid artery image capture for IMT assessment was as described for the brachial artery. 


\section{Central and peripheral $P W V$}

Pressure waves were measured using a tonometer (Millar Instruments, Houston, Texas, USA) on the right carotid and femoral arteries and via a small infrared sensor (AD Instruments, Colorado Springs, $\mathrm{CO}$, USA) on the right dorsalis pedis artery. A minimum of 10 consecutive beats were obtained. For carotid-femoral PWV (an index of central arterial stiffness), the transit distance was derived by subtracting the distance between the suprasternal notch and carotid artery from the distance between the carotid and femoral artery [(carotid femoral) - (carotid suprasternal notch)] (Weber et al. 2009). Transit distance for femoral-dorsalis pedis PWV (an index of peripheral arterial stiffness) was the distance between the two measurement points in the supine position. All distances were measured using a measuring tape held above the body surface to avoid distortion due to varying contours of the body between subjects.

\section{Data Analysis}

\section{Heart rate and Blood Pressure}

Heart rate and blood pressure during the FMD tests were analyzed offline in 3 second average time bins using the data acquisition software program LabChart (AD Instruments). Averages were calculated for the baseline of each FMD test, during exercise for HGEX-FMD and in the last minute of hyperemia for 5 and 15 min RH-FMD.

\section{Brachial Artery Blood Velocity}

Blood velocity was analyzed offline in 3 second average time bins for mean shear stress determination using the data acquisition software program LabChart (AD Instruments).

\section{Brachial Artery Diameter}

Vessel diameter was analyzed using automated edge-detection software (Encoder FMD \& Bloodflow v3.0.3, Reed Electronics, Perth, WA, Australia; an updated version of the software described in Woodman et al. (Woodman et al. 2001)) as previously described (Jazuli and Pyke 2011). The diameter 
Slattery et al., Impaired HGEX-FMD in obese males R-1

data were then compiled into 3 second average time bins. The investigator was blinded to group when performing diameter analysis.

\section{Shear Stress}

Shear stress was calculated as: $4 x$ mean blood velocity $x$ blood viscosity/vessel diameter, calculated from the 3 second average velocity and diameter time bins. For the HGEX-FMD an average of the last 9 minutes of exercise (the steady state period) was used to compare the stimulus for dilation between groups. For the RH trials the area under the curve (AUC) of the shear stress stimulus from the time of cuff release until: i) 30 seconds post release (AUC-30s) (5 min RH-FMD test) ii) 90 seconds post release (AUC-90s) (15 min RH-FMD) and iii) the time of peak diameter measurement (AUC-to-peak) (both 5 min RH-FMD and 15 min RH-FMD) was assessed.

\section{Flow-Mediated Dilation}

The \% RH-FMD was quantified by calculating the percent increase in diameter from the baseline diameter measured for 1 minute prior to cuff inflation to the peak 3 second average diameter post occlusion cuff release. In the case that artery wall tracking prior to occlusion was poor, the diameter recorded during the last minute of occlusion was used in its place (5 min RH-FMD: 1 (control) out of 56 scans; 15 min RH-FMD: 1 (obese) out of 28 scans). The \%HGEX-FMD was quantified by calculating the percent increase from the baseline diameter ( 1 minute pre-exercise baseline) to the average diameter in each minute of exercise. Absolute HGEX-FMD was calculated as the absolute difference $(\mathrm{cm})$ between the baseline and diameters same diameters used for \% FMD calculation. FMD from repeat trials (of both 5 min RH and HGEX-FMD) were averaged and treated as a single response for comparison between participants. Some scans were excluded due to poor wall tracking (HGEX: 7 (4 obese, 3 control) out of 56 scans; 5 min RH-FMD: 2 (1 obese, 1 control) out of 56 scans). In such circumstances the remaining single trial with superior wall tracking was used instead of a two trial average. One control subject was excluded from 15 min RH-FMD analysis due to exclusion of their single 15 RH-FMD trial as a result of poor wall tracking. 


\section{Shear stress stimulus-FMD response slope}

The slope of the shear stress - FMD relationship was determined for each participant using their 5 $\min \mathrm{RH}$ and $15 \min \mathrm{RH}-F M D$ tests. Slope $=[15 \min R H-F M D-5 \min R H-F M D] \div[15 \min R H-F M D$ shear stress AUC-5 min RH-FMD shear stress AUC]. This was done using both the shear stress AUCto-peak, and the AUC until a fixed time point post cuff release (5 min RH-FMD AUC-30s; 15 min RHFMD AUC-90s).

Normalized FMD

RH-FMD normalized to the shear stress stimulus was calculated as $\% F M D /$ Shear stress AUC. The following AUC were used i) AUC until the time of peak diameter measurement ( 5 min and 15 min tests) ii) 30s AUC (5 min RH test) iii) 90s AUC (15 min RH test).

\section{Common carotid artery IMT}

IMT was analyzed using Carotid Analyzer for Research (Medical Imaging Applications, Coralville, Iowa, USA). In a similar fashion to brachial artery analysis, the program allows for the selection of a region of interest and then tracks the intima and media of the artery's far wall via the intensity of the brightness of the lines. The program also allowed for the removal of erroneous data points due to tracking errors. The mean far wall IMT value from the 10s image clip was used in statistical analyses. IMT was not obtained in 4 participants ( 3 control, 1 obese) due to inadequate image quality.

\section{Central and peripheral $P W V$}

The data was band pass filtered $(\max 30 \mathrm{~Hz}$, $\min 5 \mathrm{~Hz})$ and the time elapsed between the minimum point in each cycle (indicative of the foot of the waveform) of the carotid and femoral, and the femoral and dorsalis pedis pulse waves was identified (Martin et al. 2012). PWV was calculated with the following equation: $P W V=$ distance travelled (meters) /transit time (seconds).

A minimum of 10 heart cycles was collected for analysis. A PWV value was calculated for each cycle, and then an average 10 cycle PWV was calculated for each subject. Individual cycles that differed 
Slattery et al., Impaired HGEX-FMD in obese males R-1

from the average by more than 2 SD were eliminated from the individual average calculation (all individual averages included a minimum of 8 cycles). PWV was not obtained in 2 participants ( 1 control, 1 obese) due to poor signal quality.

\section{Statistical Analysis}

T-Tests were used to compare 5 min RH-FMD, 15 min RH-FMD, the shear stress stimulus, the shear stress - FMD slope, HGEX force and participant characteristics between the two groups. For variables with a non-normal distribution a Mann-Whitney test was performed. A repeated measures analysis of variance (ANOVA) (factors: between subjects - group (obese vs. control) and within subjects - exercise time) was used analyze HGEX-FMD responses. Bonferonni corrected pairwise comparisons were applied to perform post hoc analysis on significant main effects and interactions. Due to differences in baseline diameter between groups, group comparisons of absolute FMD were also performed using allometric scaling analysis (Atkinson et al. 2013) such that log transformed baseline diameter was used as a covariate. Cohen's d was calculated for $\%$ FMD to assist in comparing the magnitude of impact of group on the three distinct FMD responses. All statistical analysis was completed using IBM SPSS, Version 20 (SPSS Inc., Chicago, IL, USA). Data was presented as means \pm SD.

\section{Results}

\section{Participant Characteristics}

The baseline characteristics of the participants are displayed in Table 1. Age, BMI, waist circumference, baseline diameter, systolic blood pressure and LDL cholesterol were significantly higher in the obese group, while HDL cholesterol was significantly lower in the obese group.

\section{$H R$ and mean arterial pressure (MAP) during FMD testing}

The HR and MAP data are reported in Table 2. Both HR and MAP increased significantly during HGEX (main effect of time $p<0.001$ ), with no differences observed between groups $(p=0.638$ and $\mathrm{p}=0.186$ for HR and MAP respectively). No changes in HR or MAP from baseline to the last minute of hyperemia were observed in the 5 min RH-FMD protocol in either group (HR: main effect of time 
$\mathrm{p}=0.176$; main effect of group $\mathrm{p}=0.878$; MAP: main effect of time $\mathrm{p}=0.978$; main effect of group $\mathrm{p}=0.404)$. In the $15 \mathrm{~min}$ RH-FMD protocol, a small but significant increase in HR was observed in the last min of hyperemia vs. baseline (main effect of time $\mathrm{p}=0.005$ ), with no differences between groups $(\mathrm{p}=0.561)$. No difference in MAP was observed between these time points (main effect of time $\mathrm{p}=0.099$ and main effect of group $\mathrm{p}=0.224)$.

\section{Shear Stress}

No differences in baseline shear stress were observed between groups in the HGEX-FMD test $(\mathrm{p}=0.417)$. The shear stress stimulus over the $10 \mathrm{~min}$ exercise bout is shown in Figure 2A. Steady state shear stress in the HGEX-FMD test was also not significantly different between groups $(\mathrm{p}=0.390)$ (Figure 2B).

In the 5 min RH-FMD test, the larger shear stress AUC-to-peak in the obese group approached significance $(\mathrm{p}=0.080)$ (Figure 2C) and in the $15 \mathrm{~min}$ RH-FMD test the AUC-to-peak was significantly larger in the obese group ( $\mathrm{p}=0.004$ ) (Figure 2D). As expected, the $15 \mathrm{~min}$ RH-FMD test AUC-to-peak was larger than in the $5 \mathrm{~min}$ RH-FMD test $(\mathrm{p}<0.001)$. When time post cuff release for shear stress AUC calculation was fixed, similar results were observed. In both the 5 min RH-FMD test and the 15 min RHFMD test the AUC-30s was significantly greater in the obese group $(\mathrm{p}=0.040)$. In the 15 min RH-FMD test the AUC-90s, was significantly greater in the obese group $(\mathrm{p}=0.015)$.

FMD

A significant effect of time $(\mathrm{p}<0.001)$, group $(\mathrm{p}=0.001)$, and a significant time by group interaction $(\mathrm{p}<0.001)$ were observed for the HGEX-FMD test (Figure 3). The obese group had a significantly impaired HGEX-FMD compared to the healthy controls (last minute of exercise: $5.2 \pm 3.0 \%$ vs. $11.5 \pm 4.4 \%$ ). The difference between the two groups became significant in the $2^{\text {nd }}$ minute of exercise and remained significantly different until the end of the exercise bout. Results for the absolute change in FMD were similar (significant effect of time $(\mathrm{p}<0.001)$; significant effect of group $(\mathrm{p}=0.001)$ and an interaction between group and time $(\mathrm{p}<0.001))$. In addition, absolute HGEX-FMD analyzed with the 
Slattery et al., Impaired HGEX-FMD in obese males R-1

allometric scaling approach also demonstrated a significant group difference $(\mathrm{p}=0.013)$. The relative (\%MVC) and absolute (represented by handgrip dynamometer voltage output) force exerted during the HGEX did not differ between groups (relative- control $47 \pm 9 \%$; obese $40 \pm 15 \%, \mathrm{p}=0.102$; absolutecontrol $0.8 \pm 0.2 \mathrm{mV}$; obese $0.7 \pm 2 \mathrm{mV} \mathrm{p}=0.298$ ).

For the 5 min RH-FMD test no group differences in \%FMD (Figure 4A), absolute FMD or timeto-peak diameter (Table 3 ) were observed $(\mathrm{p}=0.466, \mathrm{p}=0.875$ and $\mathrm{p}=0.256)$. Similarly when absolute FMD was analyzed with the allometric scaling approach no group differences were found $(p=0.269)$. Similar to the 5 min RH-FMD test, for the 15 min RH-FMD test, there were no significant group differences in \%FMD or absolute FMD or FMD analyzed with the allometric scaling approach $(\mathrm{p}=0.181$, $\mathrm{p}=0.534$ and $\mathrm{p}=0.531$ ) (Figure 4B). However, a significant group difference in the time-to-peak diameter was observed for the 15 min RH-FMD test ( $\mathrm{p}=0.026$ ) (Table 3), with the obese group taking significantly longer to reach peak diameter. The mean differences in \% FMD between groups (5 min RH-FMD: 0.9\%; 15 min RH-FMD: 3.4\%; HGEX-FMD: 6.4\%) and the Cohen's d estimate of effect size (5 min RH-FMD 0.3; 15 min RH-FMD 0.6, HGEX-FMD 1.6) support a most prominent group difference in the HGEXFMD test.

Accounting for the shear stress stimulus via ratio normalization did not reveal group differences in 5 min RH-FMD (\% 5 min RH-FMD normalized to AUC-30 p=0.118; similar results were found with absolute FMD and AUC-to-peak). In contrast, for 15 min RH-FMD ratio normalization revealed group differences ( $\% 15$ min RH-FMD normalized to AUC-90 p=0.002; similar results were found with absolute FMD and AUC-to-peak).

Shear stress stimulus-FMD response slope (RH-FMD tests)

When the shear stress was characterized as the AUC-to-peak and the response was characterized as a $\% \mathrm{FMD}$, the stimulus response slope in the obese group $(0.004 \pm 0.002)$ was significantly lower than the slope of the control group $(0.009 \pm 0.005)(\mathrm{p}=0.001)$. This remained significant when the absolute FMD was used $(\mathrm{p}=0.002)$, and when the stimulus was calculated as an AUC to a fixed time point (AUC- 
Slattery et al., Impaired HGEX-FMD in obese males R-1

30s for 5 min RH-FMD and AUC-90s for 15 min RH-FMD) with the response characterized as either a $\%$ FMD ( $\mathrm{p}=0.001)$ (Fig. 5), or an absolute FMD ( $\mathrm{p}=0.001)$. Figure 5 presents the dose response relationship for each participant and the relationship constructed from the group average data.

\section{$P W V$ and IMT}

There were no significant group differences in peripheral PWV (control $6.84 \pm 0.93 \mathrm{~m} / \mathrm{s} \mathrm{vs}$. obese $6.52 \pm 1.27 \mathrm{~m} / \mathrm{s}, \mathrm{p}=0.472$ ). The obese group had a higher central PWV (control $5.20 \pm 0.61 \mathrm{~m} / \mathrm{s}$ vs. obese $5.72 \pm 0.78 \mathrm{~m} / \mathrm{s}, \mathrm{p}=0.075$ ) and a larger carotid artery IMT (control $0.39 \pm 0.05 \mathrm{~cm}$ vs. obese $0.44 \pm 0.07$ $\mathrm{cm}, \mathrm{p}=0.075)$ which approached significance.

\section{Discussion}

This study was designed to investigate whether obesity is associated with a universal, or a shear stress profile specific impact on FMD. The key novel findings are as follows: 1) we have shown for the first time that compared to lean controls, young men with obesity had an impaired FMD response to a sustained, handgrip exercise induced shear stress stimulus, 2) this was observed alongside an absence of group differences in RH-FMD (following both a 5 and 15 min occlusion), 3) after accounting for the magnitude of the stimulus, the 15 min RH-FMD, but not the 5 min RH-FMD was significantly lower in the obese group. As a whole these findings suggest that the obese group had an impaired ability to respond to prolonged shear stress stimuli, resulting from handgrip exercise and the release of prolonged forearm occlusion, but a relatively preserved ability to respond to the brief shear stress stimulus resulting from the release of a $5 \mathrm{~min}$ forearm occlusion. These data indicate the presence of some level of endothelial dysfunction in the obese group that would be missed by assessment with a standard 5 min occlusion test alone. Although links to clinical outcomes have yet to be determined, from a physiological/functional perspective, research seeking to comprehensively evaluate brachial artery endothelial function may benefit from inclusion of FMD stimulated by prolonged increases in shear stress. We also observed a trend towards a larger carotid artery IMT and faster central PWV (approaching 
Slattery et al., Impaired HGEX-FMD in obese males R-1

significance, both $\mathrm{p}=0.075$ ). In agreement with the evidence of impaired endothelial function, this suggests that the obese group had a lower vascular health status.

\section{RH-FMD time to peak diameter}

While no differences were observed for the 5 min RH-FMD test, time to peak dilation was significantly longer in the obese group in the 15 min RH-FMD test (Table 3). This is in agreement with previous reports that a longer time to peak dilation might be indicative of vascular dysfunction (Black et al. 2008;Fernandes et al. 2014; Irace et al. 2008), and suggests that this effect may be more easily detected with a larger RH profile. Group differences in the time to peak diameter, which reflect response dynamics, could generate differences in the shear stress AUC to peak diameter measurement that do not reflect a true difference in the stimulus profile. To ensure that we had a stimulus characterization that was independent from response characteristics, we also calculated a shear stress AUC to fixed time points (30s in the 5 min RH test and 90s in the 15 min RH test). Group differences in the stimulus were similar when characterized independently from the time to peak diameter, indicating that the differences were not simply an artifact created by the longer time to peak diameter in the obese group.

\section{5 min and 15 min RH-FMD}

No significant differences in RH-FMD were detected between the obese and control groups using the 5 min RH- or 15 min RH-FMD tests. These findings are in contrast with the majority of previous studies which have observed an impaired 5 min RH-FMD response in participants with obesity (Arcaro et al. 1999;Brook et al. 2001;Hashimoto et al. 1998;Olson et al. 2006; Woo et al. 2004). Overall the RH shear stress AUC was larger in the obese group in the present study, and we considered the possibility that a higher shear stress may have masked an impaired RH-FMD in the obese group.

Although ratio normalization has some limitations and interpretation is cautioned as potentially misleading if a number of statistical assumptions are not met (including a linear relationship between shear and FMD with a zero intercept, normal distribution and homogeneous variance) (Atkinson et al. 2009; Thijssen et al. 2011), we used this tool to further explore whether the higher shear stress stimulus in 
the obese group was masking impaired RH-FMD responses. The normalized $15 \mathrm{~min}$ RH-FMD response was significantly lower in the obese group vs. the control group, while the 5 min RH-FMD still was not. The presence of a group difference in 15 min RH-FMD but not 5 min RH-FMD after accounting for stimulus magnitude was also supported by analysis of RH-FMD with shear stress AUC as a covariate (data not shown). This, in combination with the clearly impaired HGEX-FMD (Figure 3) (stimulus matched between groups (Figure 2B)) suggests that at least in this young healthy phenotype (table one blood pressure and metabolic parameters in normal range), obesity may preferentially impair the endothelium's ability to respond to more prolonged increases in shear stress.

The slope of RH-FMD - shear stress stimulus relationship was lower in the obese group. A lower slope in participants with CVD risk has been found previously by creating a range of stimuli with cuff inflation durations ranging from $1-5 \mathrm{~min}$ (Padilla et al. 2009). The current work differs from those findings by demonstrating group differences using a relationship constructed with shear stress stimuli resulting from $>5$ min of forearm occlusion, and thus into a larger stimulus-response range. Again, given the normalized RH-FMD findings (different only in the 15min RH-FMD test) and the HGEX-FMD findings, this group difference in slope was likely primarily driven by an impaired response to the prolonged hyperemia in the 15 min RH-FMD test.

\section{HGEX-FMD}

The HGEX-FMD impairment was present from the $2^{\text {nd }}$ until the $10^{\text {th }}$ minute of the exercise bout. This finding is in agreement with a recent study by our group in which we observed impaired HGEXFMD in young healthy smokers (Findlay et al. 2013). Findlay et al. (2013) also observed no impairment in 5 min RH-FMD. In addition to the present findings and those of Findlay et al. (2013) a small collection of additional studies provide evidence that FMD in response to a sustained stimulus is impaired in conditions associated with a poor cardiovascular prognosis (smokers (Gaenzer et al. 2001), the elderly (Grzelak et al. 2010), patients with type 1 diabetes (Bellien et al. 2010;Grzelak et al. 2010)), in some cases to a greater extent than RH-FMD (Bellien et al. 2010; Grzelak et al. 2010). However, this finding is 
Slattery et al., Impaired HGEX-FMD in obese males R-1

not congruent with all existing literature. Mullen et al. (2001) observed an impaired 5 min RH-FMD in patients with high cholesterol, but no impairment in FMD in response to a sustained shear stress stimulus achieved with distal vasodilator infusion.

There is evidence to suggest that different shear stress profiles recruit distinct endothelial transduction and dilatory mechanisms (Frangos et al. 1996;Mullen et al. 2001;Pyke and Tschakovsky 2005). Health condition- and shear stress profile-specific FMD results may indicate that some shear stress transduction pathways are more vulnerable to interference from particular vascular 'insults' or disease states. While both RH-FMD and FMD in response to sustained stimuli like HGEX are thought to be somewhat dependent on NO (Bellien et al. 2006) (Joannides et al. 1995; Wray et al. 2011) it is also possible that the specific pathway of NO production depends on the shear stress stimulus employed (Frangos et al. 1996). A more pronounced HGEX-FMD and 15 min RH-FMD impairment may reflect a greater negative impact of obesity on the mechanisms responsible for transduction of prolonged stimuli.

\section{The influence of baseline diameter}

Baseline diameter was larger in the obese group and this deserves consideration when interpreting the FMD responses given that baseline diameter could influence \%FMD both via the calculation itself and potentially, an obesity independent reduction in shear stress sensitivity with a larger brachial artery diameter (Thijssen et al. 2008). Furthermore, some studies have suggested that baseline diameter itself predicts cardiovascular risk, with larger diameters associated with greater risk (Yeboah et al. 2007). However, purely diameter driven sensitivity differences as an explanation for the observed group difference in HGEX-FMD is unlikely as evidence suggests that when shear stress is controlled, baseline diameter does not influence FMD in the brachial artery (Jazuli and Pyke 2011). In addition, while group differences in baseline diameter were present for all three FMD tests, group differences in FMD were found for HGEX-FMD only. To explore the influence of baseline diameter, an allometric scaling approach was performed with the absolute FMD as the outcome and log transformed baseline diameter as a covariate (Atkinson et al. 2013). In this analysis, again, group differences were found only for the 
Slattery et al., Impaired HGEX-FMD in obese males R-1

HGEX-FMD test. Taken together these data suggest that differences in baseline diameter were unlikely to be responsible our observed group differences in HGEX-FMD.

\section{Subject characteristics and mechanisms of endothelial function impairment}

The BMI and waist circumference of the obese group in the present study is slightly larger than previous studies that have detected attenuated 5 min RH-FMD in obese populations (Biasucci et al. 2010; Brook et al. 2001; Hashimoto et al. 1998). This suggests that a lower 'severity' of obesity does not explain our disparate results. The obese group presented with significantly lower HDL levels, higher blood pressure, and were older than the control group (table 1). The blood pressure in the obese group remained in the 'normal' range although HDL was below the recommended cut point (Chobanian et al. 2003; Cleeman et al. 2001). It is unlikely that mild dyslipidemia fully accounts for the development of endothelial dysfunction in the obese group. Given the modest difference in metabolic variables it is likely that other obesity related factors were involved (Lau 2005; Montero et al. 2012; Van Gaal et al. 2006). Although the obese group was significantly older than the control group ( 27 vs. 21 years) it is unlikely that age played a significant role in determining FMD. Celermajer and colleagues (Celermajer et al. 1994) observed that \%FMD (measured using the standard 5 min RH-FMD protocol) did not exhibit an age related decline in men until the $4^{\text {th }}$ decade. In addition, the group difference in HGEX-FMD persisted with age, systolic blood pressure and HDL added as a covariates (data not shown).

\section{Limitations}

This study population was limited to young, healthy men and examined the brachial artery only, which therefore limits its generalizability to other age groups, to women and to other vascular beds. Future studies are required to investigate whether the obesity-mediated inhibition of the dilatory response to a sustained shear stress stimulus is sex, age and vessel specific.

Oxidative stress and indices of inflammation were not assessed, therefore we were not able to evaluate whether these specific characteristics in the obese group might have influenced the FMD responses observed. However, these measurements would not have aided in answering the primary 
Slattery et al., Impaired HGEX-FMD in obese males R-1

question, which was to determine whether the presence of obesity is associated with a uniform, or a shear stress profile specific impact on FMD. In addition, with the non-invasive design we were not able to identify the specific mechanistic etiology of the distinct RH and HGEX-FMD responses. Finally, with the inclusion of only one abdominally obese group without visceral adipose tissue quantification, the findings do not provide insight regarding the sensitivity of HGEX-FMD in detecting dysfunction over a range of phenotypes of adiposity. Future, larger studies will be required to address this limitation.

\section{Conclusion}

This study is the first to investigate how obesity affects the ability of the brachial artery to respond to various shear stress profiles. With a matched shear stress stimulus, it was shown for the first time that HGEX-FMD was more than 50\% lower in young males with abdominal obesity vs. a lean control group. We also showed for the first time a lower normalized 15 min RH-FMD, and shear stress stimulus-RH-FMD response slope in the obese group. Taken together these data suggest that obesity is associated with impairment the endothelium's ability to respond to prolonged increases in shear stress. Future work is needed to establish: 1) the sensitivity of HGEX-FMD in detecting endothelial dysfunction in a range of phenotypes of excess adiposity and other conditions, 2) The mechanisms through which obesity causes impairments in FMD and 3) whether the HGEX-FMD and 15 min RH-FMD are useful in the prediction of future cardiovascular risk.

\section{CONFLICT OF INTEREST}

The authors have no conflicts to report.

\section{AKNOWLEGEMENTS}

This study was funded by a Natural Sciences and Engineering Research Council of Canada Discovery Grant and Canada Foundation for Innovation and Ontario Ministry of Research and Innovation Leaders Opportunity Funding to K.E.Pyke. D.J. Slattery and T.J.R. Stuckless were supported by a Ministry of Research Innovation Young Researcher Award to K.E.Pyke. T.J. King was supported by a Natural Sciences and Engineering Research Council of Canada PGS scholarship. 


\section{Reference List}

Arcaro, G., Zamboni, M., Rossi, L., Turcato, E., Covi, G., Armellini, F.et al. 1999. Body fat distribution predicts the degree of endothelial dysfunction in uncomplicated obesity. International journal of obesity and related metabolic disorders : journal of the International Association for the Study of Obesity. 23(9): 936-942.

Atkinson, G., Batterham, A. M., Black, M. A., Cable, N. T., Hopkins, N. D., Dawson, E. A.et al. 2009. Is the ratio of flow-mediated dilation and shear rate a statistically sound approach to normalization in crosssectional studies on endothelial function? J Appl Physiol. 107(6): 1893-1899.

Atkinson, G., Batterham, A. M., Thijssen, D. H., and Green, D. J. 2013. A new approach to improve the specificity of flow-mediated dilation for indicating endothelial function in cardiovascular research. $\mathrm{J}$ Hypertens. 31(2): 287-291.

Bellien, J., Costentin, A., Dutheil-Maillochaud, B., Iacob, M., Kuhn, J. M., Thuillez, C.et al. 2010. Early stage detection of conduit artery endothelial dysfunction in patients with type 1 diabetes. Diab Vasc Dis Res. 7(2): 158-166.

Bellien, J., Iacob, M., Gutierrez, L., Isabelle, M., Lahary, A., Thuillez, C.et al. 2006. Crucial role of NO and endothelium-derived hyperpolarizing factor in human sustained conduit artery flow-mediated dilatation. Hypertension. 48(6): 1088-1094.

Biasucci, L. M., Graziani, F., Rizzello, V., Liuzzo, G., Guidone, C., De Caterina, A. R.et al. 2010. Paradoxical Preservation of Vascular Function in Severe Obesity. The American Journal of Medicine. 123(8): 727-734.

Black, M. A., Cable, N. T., Thijssen, D. H., and Green, D. J. 2008. Importance of measuring the time course of flow-mediated dilatation in humans. Hypertension. 51(2): 203-210.

Brook, R. D., Bard, R. L., Rubenfire, M., Ridker, P. M., and Rajagopalan, S. 2001. Usefulness of visceral obesity (waist/hip ratio) in predicting vascular endothelial function in healthy overweight adults. The American journal of cardiology. 88(11): 1264-1269.

Celermajer, D. S., Sorensen, K. E., Gooch, V. M., Spiegelhalter, D. J., Miller, O. I., Sullivan, I. D.et al. 1992. Non-invasive detection of endothelial dysfunction in children and adults at risk of atherosclerosis. Lancet. 340(8828): 1111-1115. 
Slattery et al., Impaired HGEX-FMD in obese males R-1

Celermajer, D. S., Sorensen, K. E., Spiegelhalter, D. J., Georgakopoulos, D., Robinson, J., and Deanfield, J. E. 1994. Aging is associated with endothelial dysfunction in healthy men years before the age-related decline in women. J Am Coll Cardiol. 24(2): 471-476.

Chobanian, A. V., Bakris, G. L., and Black, H. R. 2003. The seventh report of the joint national committee on prevention, detection, evaluation, and treatment of high blood pressure: The jnc 7 report. JAMA. 289(19): 2560-2571.

Cleeman, J. I., Grundy, S. M., Becker, D., and Clark, L. T. 2001. Expert panel on Detection, Evaluation and Treatment of High blood Cholesterol in Adults. Executive Summary of the Third Report of the National Cholesterol Education Program (NCEP) Adult Treatment Panel (ATP III). JAMA. 285(19): 2486-2497.

Deanfield, J. E., Halcox, J. P., and Rabelink, T. J. 2007. Endothelial function and dysfunction: testing and clinical relevance. Circulation. 115(10): 1285-1295.

Duffy, S. J., Castle, S. F., Harper, R. W., and Meredith, I. T. 1999. Contribution of vasodilator prostanoids and nitric oxide to resting flow, metabolic vasodilation, and flow-mediated dilation in human coronary circulation. Circulation. 100(19): 1951-1957.

Fernandes, I. A., Sales, A. R. K., Rocha, N. G., Silva, B. M., Vianna, L. C., and da Nóbrega, A. C. L. 2014. Preserved flow-mediated dilation but delayed time-to-peak diameter in individuals with metabolic syndrome. Clin Physiol Funct Imaging. 34(4): 270-276.

Findlay, B. B., Gupta, P., Szijgyarto, I. C., and Pyke, K. E. 2013. Impaired brachial artery flow-mediated vasodilation in response to handgrip exercise-induced increases in shear stress in young smokers. Vasc Med. 18(2): 63-71.

Frangos, J. A., Huang, T. Y., and Clark, C. B. 1996. Steady shear and step changes in shear stimulate endothelium via independent mechanisms--superposition of transient and sustained nitric oxide production. Biochem Biophys Res Commun. 224(3): 660-665.

Gaenzer, H., Neumayr, G., Marschang, P., Sturm, W., Kirchmair, R., and Patsch, J. R. 2001. Flowmediated vasodilation of the femoral and brachial artery induced by exercise in healthy nonsmoking and smoking men. J Am Coll Cardiol. 38(5): 1313-1319.

Grzelak, P., Olszycki, M., Majos, A., Czupryniak, L., Strzelczyk, J., and Stefanczyk, L. 2010. Hand exercise test for the assessment of endothelium-dependent vasodilatation in subjects with type 1 diabetes. Diabetes Technol Ther. 12(8): 605-611.

Han, T. S. 1995. Waist circumference action levels in the identification of cardiovascular risk factors: prevalence study in a random sample. BMJ. 311(7017): 1401-1405. 
Harris, R. A., Padilla, J., Rink, L. D., and Wallace, J. P. 2006. Variability of flow-mediated dilation measurements with repetitive reactive hyperemia. Vasc Med. 11(1): 1-6.

Hashimoto, M., Akishita, M., Eto, M., Kozaki, K., Ako, J., Sugimoto, N.et al. 1998. The impairment of flow-mediated vasodilatation in obese men with visceral fat accumulation. International journal of obesity and related metabolic disorders: journal of the International Association for the Study of Obesity. 22(5): 477-484.

Inaba, Y., Chen, J. A., and Bergmann, S. R. 2010. Prediction of future cardiovascular outcomes by flowmediated vasodilatation of brachial artery: a meta-analysis. Int J Cardiovasc Imaging. 26(6): 631-640.

Irace, C., Tschakovsky, M. E., Carallo, C., Cortese, C., and Gnasso, A. 2008. Endothelial dysfunction or dysfunctions? Identification of three different FMD responses in males with type 2 diabetes.

Atherosclerosis. 200(2): 439-445.

Jazuli, F. and Pyke, K. E. 2011. The impact of baseline artery diameter on flow-mediated vasodilation: a comparison of brachial and radial artery responses to matched levels of shear stress. Am J Physiol Heart Circ Physiol. 301(4): H1667-H1677.

Joannides, R., Haefeli, W. E., Linder, L., Richard, V., Bakkali, E. H., Thuillez, C.et al. 1995. Nitric oxide is responsible for flow-dependent dilatation of human peripheral conduit arteries in vivo. Circulation.

91(5): 1314-1319.

King, T. J., Slattery, D. J., and Pyke, K. E. 2013. The impact of handgrip exercise duty cycle on brachial artery flow-mediated dilation. Eur J Appl Physiol. 113(7): 1849-1858.

$\mathrm{Ku}$, J., McEvoy, A., and Pyke, K. E. 2014. Can a combination of handgrip exercise and prolonged forearm occlusion elicit a maximal brachial artery FMD response? Eur J Appl Physiol. 114(6): 12971307.

Lau, D. C. W. D. 2005. Adipokines: molecular links between obesity and atheroslcerosis. American Journal of Physiology - Heart and Circulatory Physiology. 288(5): H2031-H2041.

Martin, A. A., Cotie, L. M., Timmons, B. W., Gorter, J. W., and MacDonald, M. J. 2012. Arterial structure and function in ambulatory adolescents with cerebral palsy are not different from healthy controls. Int J Pediatr. 2012: 168209.

Meyer, A. A., Kundt, G., Steiner, M., Schuff-Werner, P., and Kienast, W. 2006. Impaired flow-mediated vasodilation, carotid artery intima-media thickening, and elevated endothelial plasma markers in obese children: the impact of cardiovascular risk factors. Pediatrics. 117(5): 1560-1567. 
Slattery et al., Impaired HGEX-FMD in obese males R-1

Montero, D., Walther, G., Perez-Martin, A., Roche, E., and Vinet, A. 2012. Endothelial dysfunction, inflammation, and oxidative stress in obese children and adolescents: markers and effect of lifestyle intervention. Obesity Reviews. 13(5): 441-455.

Mullen, M. J., Kharbanda, R. K., Cross, J., Donald, A. E., Taylor, M., Vallance, P.et al. 2001. Heterogenous nature of flow-mediated dilatation in human conduit arteries in vivo: relevance to endothelial dysfunction in hypercholesterolemia. Circ Res. 88(2): 145-151.

Nabel, E. G., Selwyn, A. P., and Ganz, P. 1990. Large coronary arteries in humans are responsive to changing blood flow: an endothelium-dependent mechanism that fails in patients with atherosclerosis. J Am Coll Cardiol. 16(2): 349-356.

Olson, T. P., Schmitz, K. H., Leon, A. S., and Dengel, D. R. 2006. Vascular Structure and Function in Women: Relationship with Body Mass Index. American Journal of Preventive Medicine. 30(6): 487-492.

Padilla, J., Harris, R. A., Fly, A. D., Rink, L. D., and Wallace, J. P. 2006. A comparison between activeand reactive-hyperaemia-induced brachial artery vasodilation. Clin Sci (Lond). 110(3): 387-392.

Padilla, J., Johnson, B. D., Newcomer, S. C., Wilhite, D. P., Mickleborough, T. D., Fly, A. D.et al. 2009. Adjusting flow-mediated dilation for shear stress stimulus allows demonstration of endothelial dysfunction in a population with moderate cardiovascular risk. J Vasc Res. 46(6): 592-600.

Pyke, K. E., Hartnett, J. A., and Tschakovsky, M. E. 2008a. Are the dynamic response characteristics of brachial artery flow mediated dilation sensitive to the magnitude of increase in shear stimulus? J Appl Physiol. 105(1): 282-292.

Pyke, K. E. and Jazuli, F. 2011. Impact of repeated increases in shear stress via reactive hyperemia and handgrip exercise: no evidence of systematic changes in brachial artery FMD. Am J Physiol Heart Circ Physiol. 300(3): H1078-H1089.

Pyke, K. E., Poitras, V., and Tschakovsky, M. E. 2008b. Brachial artery flow mediated dilation during handgrip exercise: evidence for endothelial transduction of the mean shear stimulus. Am J Physiol Heart Circ Physiol. 294(6): H2669-H2679.

Pyke, K. E. and Tschakovsky, M. E. 2005. The relationship between shear stress and flow-mediated dilatation: implications for the assessment of endothelial function. J Physiol. 568(Pt 2): 357-369.

Szijgyarto, I. C., King, T. J., Ku, J., Poitras, V. J., Gurd, B. J., and Pyke, K. E. 2013. The impact of acute mental stress on brachial artery flow-mediated dilation differs when shear stress is elevated by reactive hyperemia versus handgrip exercise. Appl Physiol Nutr Metab. 38(5): 498-506. 
Thijssen, D. H., Black, M. A., Pyke, K. E., Padilla, J., Atkinson, G., Harris, R. A.et al. 2011. Assessment of flow-mediated dilation in humans: a methodological and physiological guideline. Am J Physiol Heart Circ Physiol. 300(1): H2-12.

Thijssen, D. H., Dawson, E. A., Black, M. A., Hopman, M. T., Cable, N. T., and Green, D. J. 2008. Heterogeneity in conduit artery function in humans: impact of arterial size. Am J Physiol Heart Circ Physiol.

Van Gaal, L. F., Mertens, I. L., and De Block, C. E. 2006. Mechanisms linking obesity with cardiovascular disease. Nature. 444(7121): 875-880.

Vita, J. A. and Hamburg, N. M. 2010. Does endothelial dysfunction contribute to the clinical status of patients with peripheral arterial disease? Can J Cardiol. 26 Suppl A: 45A-50A.

Weber, T., Ammer, M., Rammer, M., Adji, A., O'Rourke, M. F., Wassertheurer, S.et al. 2009. Noninvasive determination of carotid-femoral pulse wave velocity depends critically on assessment of travel distance: a comparison with invasive measurement. J Hypertens. 27(8): 1624-1630.

Woo, K. S., Chook, P., Yu, C. W., Sung, R. Y. T., Qiao, M., Leung, S. S. F.et al. 2004. Overweight in children is associated with arterial endothelial dysfunction and intima-media thickening. International journal of obesity and related metabolic disorders : journal of the International Association for the Study of Obesity. 28(7): 852-857.

Woodman, R. J., Playford, D. A., Watts, G. F., Cheetham, C., Reed, C., Taylor, R. R.et al. 2001. Improved analysis of brachial artery ultrasound using a novel edge-detection software system. J Appl Physiol. 91(2): 929-937.

Wray, D. W., Uberoi, A., Lawrenson, L., and Richardson, R. S. 2006. Evidence of preserved endothelial function and vascular plasticity with age. Am J Physiol Heart Circ Physiol. 290(3): H1271-H1277.

Wray, D. W., Witman, M. A., Ives, S. J., McDaniel, J., Fjeldstad, A. S., Trinity, J. D.et al. 2011. Progressive handgrip exercise: evidence of nitric oxide-dependent vasodilation and blood flow regulation in humans. Am J Physiol Heart Circ Physiol. 300(3): H1 101-H1107.

Yeboah, J., Crouse, J. R., Hsu, F. C., Burke, G. L., and Herrington, D. M. 2007. Brachial flow-mediated dilation predicts incident cardiovascular events in older adults: the Cardiovascular Health Study.

Circulation. 115(18): 2390-2397. 
Slattery et al., Impaired HGEX-FMD in obese males R-1

Table 1. Participant characteristics

\begin{tabular}{lccc}
\hline & Obese & Control & $\mathrm{p}$-value \\
\hline Age (years) & $27 \pm 6$ & $21 \pm 2$ & $\mathrm{p}=0.016$ \\
BMI (kg/m²) & $36.1 \pm 6.9$ & $22.5 \pm 2.5$ & $\mathrm{p}<0.001$ \\
Waist Circumference (cm) & $115.2 \pm 11.7$ & $76.0 \pm 6.2$ & $\mathrm{p}<0.001$ \\
Brachial artery diameter HGEX (cm) & $0.39 \pm 0.04$ & $0.34 \pm 0.04$ & $\mathrm{p}=0.006$ \\
Brachial artery diameter 5 RH (cm) & $0.39 \pm 0.04$ & $0.34 \pm 0.04$ & $\mathrm{p}=0.004$ \\
Brachial artery diameter 15 RH (cm) & $0.39 \pm 0.04$ & $0.34 \pm 0.04$ & $\mathrm{p}=0.006$ \\
Blood Viscosity (centipois) & $4.93 \pm 0.76$ & $4.33 \pm 0.41$ & $\mathrm{p}=0.090$ \\
Systolic Blood Pressure (mmHg) & $114 \pm 10$ & $104 \pm 7$ & $\mathrm{p}=0.009$ \\
Diastolic Blood Pressure (mmHg) & $71 \pm 11$ & $64 \pm 8$ & $\mathrm{p}=0.057$ \\
Physical Activity (kcal/kg/week) & $258 \pm 70$ & $235 \pm 10$ & $\mathrm{p}=0.980$ \\
HDL (mg/dL) & $36 \pm 7$ & $50 \pm 15$ & $\mathrm{p}=0.005$ \\
LDL (mg/dL) & $111 \pm 37$ & $88 \pm 35$ & $\mathrm{p}=0.213$ \\
Glucose (mg/dL) & $86 \pm 9$ & $94 \pm 16$ & $\mathrm{p}=0.153$ \\
\hline
\end{tabular}

Table 1. Values are means \pm SD. BMI- body mass index; HGEX- handgrip exercise; RH- reactive hyperemia; HDL- high density lipoprotein; LDL- low density lipoprotein. LDL: obese $n=8$, control n=9; Blood Viscosity: obese: $n=7$, control $n=18$. (values for $n<19$ occurred when a blood draw could not be obtained, or when values were outside of the range detected by our device). 
Table 2. Hemodynamic parameters during the FMD tests

\begin{tabular}{|c|c|c|c|c|}
\hline & & & \multicolumn{2}{|c|}{ Group } \\
\hline FMD test & Parameter & Time & Obese & control \\
\hline \multirow[t]{4}{*}{ HGEX-FMD } & HR & Baseline & $61 \pm 10$ & $60 \pm 7$ \\
\hline & & Exercise* & $68 \pm 6$ & $67 \pm 7$ \\
\hline & MAP & Baseline & $102 \pm 6$ & $97 \pm 8$ \\
\hline & & Exercise* & $109 \pm 7$ & $105 \pm 9$ \\
\hline \multirow[t]{4}{*}{ 5RH-FMD } & HR & Baseline & $61 \pm 7$ & $60 \pm 12$ \\
\hline & & $\begin{array}{l}\text { Last min of } \\
\text { hyperemia }\end{array}$ & $61 \pm 7$ & $61 \pm 12$ \\
\hline & MAP & Baseline & $99 \pm 10$ & $96 \pm 10$ \\
\hline & & $\begin{array}{l}\text { Last min of } \\
\text { hyperemia }\end{array}$ & $99 \pm 7$ & $96 \pm 10$ \\
\hline \multirow[t]{4}{*}{15 RH-FMD } & HR & Baseline & $59 \pm 7$ & $58 \pm 7$ \\
\hline & & $\begin{array}{l}\text { Last min of } \\
\text { hyperemia* }\end{array}$ & $61 \pm 6$ & $60 \pm 6$ \\
\hline & MAP & Baseline & $104 \pm 6$ & $100 \pm 7$ \\
\hline & & $\begin{array}{l}\text { Last min of } \\
\text { hyperemia }\end{array}$ & $106 \pm 10$ & $103 \pm 9$ \\
\hline
\end{tabular}

Heart rate (HR) and Mean arterial pressure (MAP) during flow mediated dilation (FMD) tests. Values are means $\pm \mathrm{SD}$. * significantly different from the baseline condition within that test. HGEX-FMD-handgrip exercise flow mediated dilation; RH-FMD- reactive hyperemia flow mediated dilation. 
Slattery et al., Impaired HGEX-FMD in obese males R-1

Table 3. Time to peak diameter measurement

\begin{tabular}{cccc}
\hline & Obese & Control & p-value \\
\hline Time-to-peak (s) (5 min RH-FMD) & $48 \pm 16$ & $43 \pm 14$ & $\mathrm{p}=0.256$ \\
Time-to-peak (s) (15 min RH-FMD) & $109 \pm 20$ & $89 \pm 21$ & $\mathrm{p}=0.026$
\end{tabular}

Table 3. The time from occlusion cuff release until the time of peak diameter measurement. Values are means $\pm \mathrm{SD}$. RH-FMD-reactive hyperemia flow-mediated dilation. 


\section{Figure Legends}

Figure 1. Protocol timeline. The order of the handgrip exercise (HGEX) and 5 min reactive hyperemia (RH) flow-mediated dilation (FMD) tests was counterbalanced between participants. The * denotes the time of a venous blood draw for blood viscosity, glucose and lipid determination.

Figure 2. Panel A- HGEX-FMD shear stress - one minute of baseline and 10 minutes of exercise are presented. Filled circles are obese participants and open circles are controls. Panel B- The steady state HGEX shear stress stimulus (last 9 min of exercise). Panel C- 5 min RH-FMD AUC to peak diameter measurement. Panel D- 15 min RH-FMD AUC to peak diameter measurement. \# represents a significant group difference $(\mathrm{p}<0.05)$. AUC- Area under the curve. HGEX-FMDHandgrip exercise flow-mediated dilation. RH-FMD -reactive hyperemia flow-mediated dilation. Error bars represent the SD.

Figure 3. \%HGEX-FMD; one minute of baseline and 10 minutes of exercise are presented. * indicates a significant difference between controls and obese participants from the $2^{\text {nd }}$ minute to the $10^{\text {th }}$ minute $(\mathrm{p}<0.005)$. HGEX-FMD- Handgrip exercise flow-mediated dilation. Error bars represent the SD.

Figure 4. Panel A- \%5 min RH-FMD; Panel B- \%15 min RH-FMD. RH-FMD -reactive hyperemia flow-mediated dilation. Error bars represent the SD 
Figure 5. The shear stress stimulus-RH-FMD response relationship. Thin solid lines and filled circles present the individual slopes in the control group and dashed lines and open circles present the individual slopes in the obese group. The bold lines and squares represent the group average data. The shear stress is characterized as the AUC to $30 \mathrm{~s}$ ( 5 min RH-FMD test) and 90s ((15 min RH-FMD test). AUC- area under the curve. RH-FMD -reactive hyperemia flowmediated dilation. Error bars represent the SD. 


\section{Figure 1}

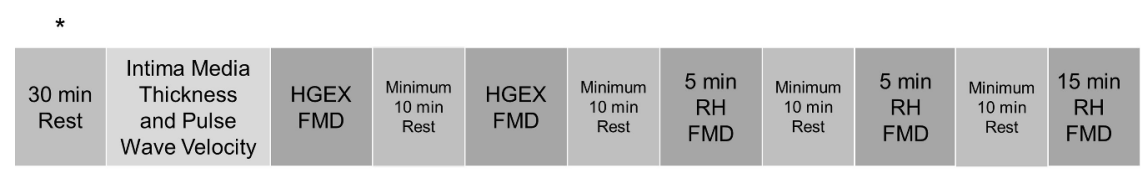

\begin{tabular}{c|c|c|c|}
$\begin{array}{c}5 \text { and } 15 \mathrm{~min} \\
\text { RH-FMD } \\
\text { Protocol: }\end{array}$ & $\begin{array}{c}1 \text { minute } \\
\text { of } \\
\text { Baseline }\end{array}$ & $\begin{array}{c}5 \text { or } 15 \text { minutes of } \\
\text { cuff occlusion }(250 \\
\mathrm{mmHg})\end{array}$ & $\begin{array}{c}3 \text { minutes of post } \\
\text { cuff release } \\
\text { recording }\end{array}$ \\
$\begin{array}{c}\text { HGEX-FMD } \\
\text { Protocol: }\end{array}$ & $\begin{array}{c}1 \text { minute } \\
\text { of } \\
\text { Baseline }\end{array}$ & $\begin{array}{c}10 \text { minutes of } \\
\text { handgrip exercise }\end{array}$ & \\
\hline
\end{tabular}

Figure 1. Protocol timeline. The order of the handgrip exercise (HGEX) and 5 min reactive hyperemia (RH) flow-mediated dilation (FMD) tests was counterbalanced between participants. The $*$ denotes the time of a venous blood draw for blood viscosity, glucose and lipid determination.

$254 \times 190 \mathrm{~mm}(300 \times 300 \mathrm{DPI})$ 
Figure 2

A

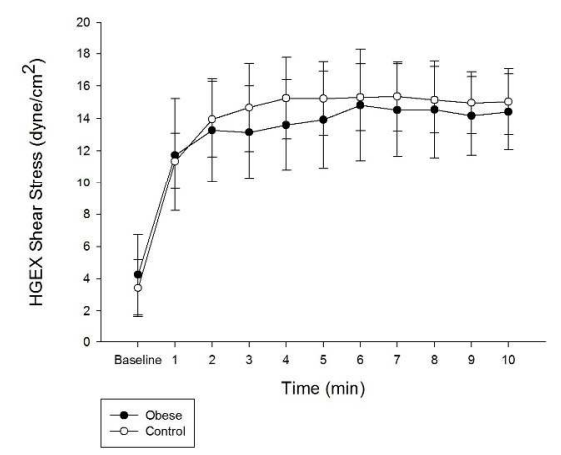

C

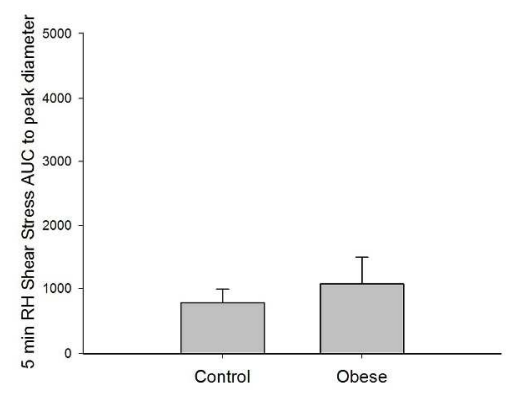

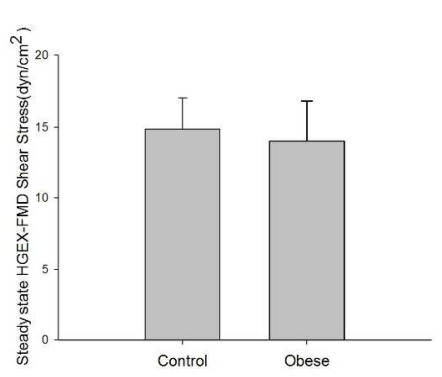

D

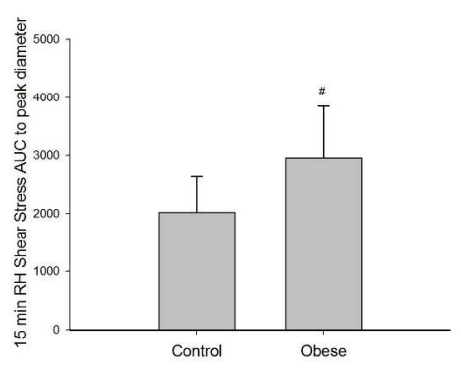

Figure 2. Panel A- HGEX-FMD shear stress - one minute of baseline and 10 minutes of exercise are presented. Filled circles are obese participants and open circles are controls. Panel B- The steady state HGEX shear stress stimulus (last 9 min of exercise). Panel C- 5 min RH-FMD AUC to peak diameter measurement. Panel D- 15 min RH-FMD AUC to peak diameter measurement. \# represents a significant group difference $(p<0.05)$. AUC- Area under the curve. HGEX-FMD- Handgrip exercise flow-mediated dilation. RH-FMD -reactive hyperemia flow-mediated dilation. Error bars represent the SD. $254 \times 190 \mathrm{~mm}(300 \times 300 \mathrm{DPI})$ 
Figure 3

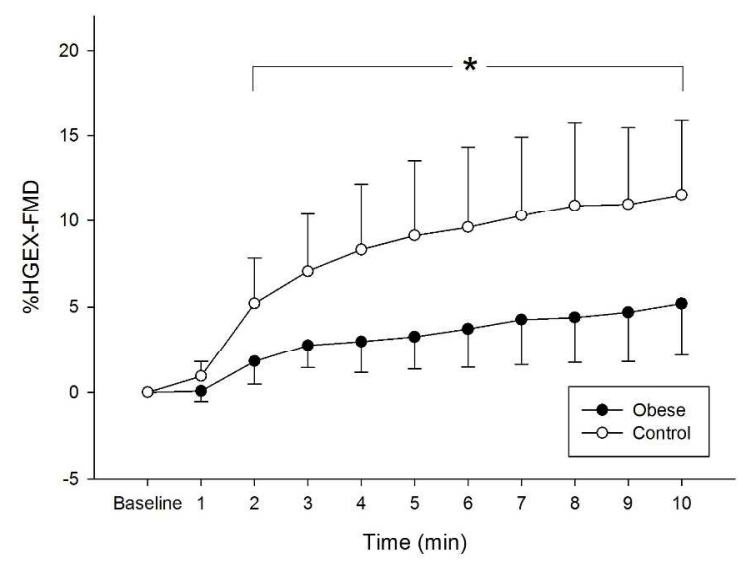

Figure 3. \%HGEX-FMD; one minute of baseline and 10 minutes of exercise are presented. * indicates a significant difference between controls and obese participants from the 2 nd minute to the 10 th minute $(p<0.005)$. HGEX-FMD- Handgrip exercise flow-mediated dilation. Error bars represent the SD.

$254 \times 190 \mathrm{~mm}(300 \times 300 \mathrm{DPI})$ 
Figure 4

A

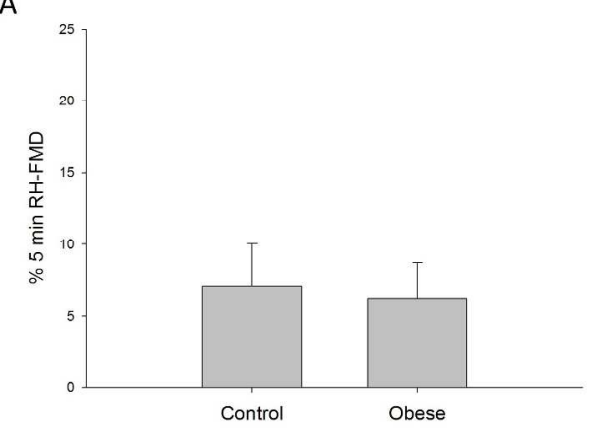

B

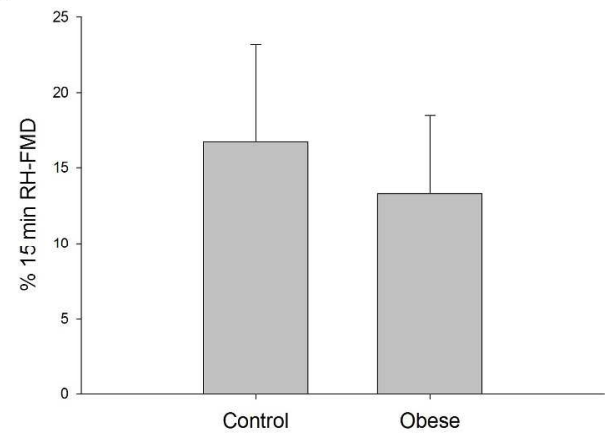

Figure 4. Panel A- \%5 min RH-FMD; Panel B- \%15 min RH-FMD. RH-FMD -reactive hyperemia flow-mediated dilation. Error bars represent the SD

$254 \times 190 \mathrm{~mm}(300 \times 300 \mathrm{DPI})$ 
Figure 5

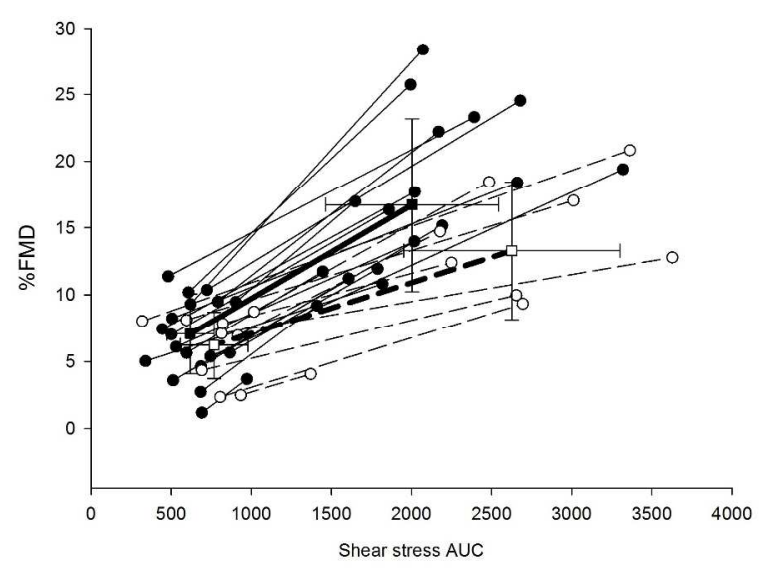

Figure 5. The shear stress stimulus-RH-FMD response relationship. Thin solid lines and filled circles present the individual slopes in the control group and dashed lines and open circles present the individual slopes in the obese group. The bold lines and squares represent the group average data. The shear stress is characterized as the AUC to 30s ( 5 min RH-FMD test) and 90s ((15 min RH-FMD test). AUC-area under the curve. RH-FMD -reactive hyperemia flow-mediated dilation. Error bars represent the SD. $254 \times 190 \mathrm{~mm}(300 \times 300 \mathrm{DPI})$ 